\title{
Evaluation of Prenatal-Onset Osteochondrodysplasias by Ultrasonography: A Retrospective and Prospective Analysis
}

\author{
Deborah Krakow, ${ }^{1,2,3 *}$ Yasemin Alanay, ${ }^{1,4}$ Lauren P. Rimoin, ${ }^{1}$ Victoria Lin, ${ }^{1}$ William R. Wilcox,${ }^{1,5}$ \\ Ralph S. Lachman, ${ }^{1,5,6}$ and David L. Rimoin ${ }^{1,2,5,7}$ \\ ${ }^{1}$ Medical Genetics Institute, Cedars-Sinai Medical Center, David Geffen School of Medicine at UCLA, Los Angeles, California \\ ${ }^{2}$ Department of Human Genetics, David Geffen School of Medicine at UCLA, Los Angeles, California \\ ${ }^{3}$ Department of Obstetrics and Gynecology, David Geffen School of Medicine at UCLA, Los Angeles, California \\ ${ }^{4}$ Department of Pediatrics, Genetics Section, Hacettepe University Faculty of Medicine, Ankara, Turkey \\ ${ }^{5}$ Department of Pediatrics, David Geffen School of Medicine at UCLA, Los Angeles, California \\ ${ }^{6}$ Department of Radiology, David Geffen School of Medicine at UCLA, Los Angeles, California \\ ${ }^{7}$ Department of Internal Medicine, David Geffen School of Medicine at UCLA, Los Angeles, California
}

Received 22 September 2007; Accepted 10 January 2008

The osteochondrodysplasias or skeletal dysplasias are a heterogenous group of over 350 distinct disorders of skeletogenesis. Many manifest in the prenatal period, making them amenable to ultrasound prenatal diagnosis. A retrospective analysis evaluated 1,500 cases referred to the International Skeletal Dysplasia Registry (ISDR) to determine the relative frequency of specific osteochondrodysplasias and correlation of ultrasound versus radiographic diagnoses for these disorders. Within the retrospective cohort of 1,500 cases, $85 \%$ of the referred cases represented well-defined skeletal dysplasias, and the other $15 \%$ of cases were a mixture of genetic syndromes and probable early-onset intrauterine growth restriction. The three most common prenatal-onset skeletal dysplasias were osteogenesis imperfecta type 2, thanatophoric dysplasia and achondrogenesis 2 , accounting for almost $40 \%$ of the cases. In a prospective analysis of 500 cases using a standardized ultrasound approach to the evaluation of these disorders, the relative frequencies of osteogenesis imperfecta type 2, thanatophoric dysplasia and achondrogenesis 2 were similar to the retrospective analysis. This study details the relative frequencies of specific prenatal-onset osteochondrodysplasias, their heterogeneity of prenatal-onset skeletal disorders and provides a standardized prenatal ultrasound approach to these disorders which should aid in the prenatal diagnosis of fetuses suspected of manifesting skeletal dysplasias.

(c) 2008 Wiley-Liss, Inc.

Key words: prenatal diagnosis; osteochondrodysplasias; skeletal dysplasias; ultrasound

How to cite this article: Krakow D, Alanay Y, Rimoin LP, Lin V, Wilcox WR, Lachman RS, Rimoin DL. 2008. Evaluation of prenatal-onset osteochondrodysplasias by ultrasonography: A retrospective and prospective analysis. Am J Med Genet Part A 146A:1917-1924.

\section{INTRODUCTION}

The osteochondrodysplasias are a heterogenous group of over 350 disorders of skeletogenesis [Hall, 2002; Superti-Furga and Unger, 2007]. They have been defined by employing a combination of clinical, radiographic, histopathologic, and molecular genetic criteria [Superti-Furga et al., 2001]. These disorders can be inherited as autosomal dominant, autosomal recessive or X-linked disorders and teratogen exposure, maternal lupus and uniparental disomy can produce phenocopies of skeletal dysplasias [Walter et al., 1996; Savarirayan, 1999; Sutton et al., 2003; Shanske et al., 2007; Superti-Furga and Unger, 2007]. Mutations in the genes responsible for these disorders affect the patterning of the skeleton, joint morphogenesis, linear growth and the integrity of the articular surface [Mundlos, 2001; Shum et al., 2003]. While the occurrence of each skeletal disorder

Grant sponsor: NIHCD; Grant number: HD2257; Grant sponsor: Joseph Drown Foundation; Grant sponsor: General Clinical Research Center; Grant number: M01-RR00425; Grant sponsor: Winnick Family Scholar awardees.

*Correspondence to: Deborah Krakow, M.D., Cedars-Sinai Medical Center, 8700 Beverly Boulevard, Medical Genetics Institute, Metro Building, Los Angeles, California 90048.

E-mail: deborah.krakow@cshs.org

DOI 10.1002/ajmg.a.32269 
is relatively rare, collectively they account for a significant number of newborns with genetic disorders, approximately 1 in 5,000 [Orioli et al., 1986].

Both the appendicular and the axial skeletons undergo a programmed pattern of endochondral ossification, whereas the calvarium and portions of the clavicle and pubis ossify via membraneous ossification [Olsen et al., 2000; Eames et al., 2003]. Ossification occurs at relatively early human gestational ages: clavicle and mandible at 8 weeks; the appendicular skeleton, ileum and scapula by 12 weeks; and the metacarpals and metatarsals are ossified by 12-16 weeks [Larson, 1993; van ZalenSprock et al., 1997]. Secondary (epiphyseal) ossification centers can be seen by radiographs by 20 weeks gestation. Since bone is echodense by ultrasound, the fetal skeleton/bone is relatively well visualized by two-dimensional ultrasound in the second trimester of pregnancy. Ultrasound evaluation as a tool for identification of congenital abnormalities has become routine in many centers throughout the world [Saltvedt et al., 2006; BahadoSingh and Raymond, 2007]. Thus, prenatal-onset skeletal dysplasias, especially the lethal disorders, are readily visualized and ideally should be diagnosed by prenatal ultrasound. The prognostic benefits of accurate diagnosis include determination of perinatal lethality, consideration for focused molecular analysis, prediction of neonatal complications, recurrence risk, and maternal management. The issue of mode of delivery in the skeletal dysplasias remains controversial [Kuller et al., 1996], though knowing whether or not the disorder is associated with macrocephaly or other abnormalities may help influence obstetrical management.

Previously published series on the diagnostic accuracy of prenatal ultrasound for the osteochondrodysplasias have noted that about $40 \%$ of these disorders are correctly diagnosed in the prenatal period [Donnenfeld and Mennuti, 1987; Sharony et al., 1993; Rasmussen et al., 1996; Gaffney et al., 1998; Tretter et al., 1998; Doray et al., 2000; Parilla et al., 2003]. Through the International Skeletal Dysplasia Registry, retrospective and prospective studies were performed to achieve the following goals: to reassess the diagnostic accuracy of prenatal ultrasound for the skeletal dysplasias, to determine the relative frequency of specific disorders, to determine which skeletal dysplasias had abnormal findings in the prenatal period and, ultimately, to determine if a standardized approach to evaluation of these fetuses could improve prenatal diagnostic accuracy.

\section{MATERIALS AND METHODS}

\section{Retrospective Analysis}

The International Skeletal Dysplasia Registry (ISDR) is a referral research registry focused on the study of osteochondrodysplasias and dysostoses. For each registered case the following information is obtained; referring diagnosis, clinical information, radiographs and, in some cases, tissues for histologic, ultrastructural, biochemical and molecular analysis. The ISDR team analyses the clinical information, radiographs and histology to determine the diagnosis (authors, DK, DLR, WRW, and RSL). The database from 1990 to 2004 was searched for cases in which the propositus was a fetus or a neonate ( $<30$ days of age). Only cases with the following features were utilized: propositus ascertained between 14 weeks gestation and one month neonatal age; at least one prenatal sonogram was performed during gestation and a referring diagnosis was based on sonographic findings; and postnatal radiographs and available histology were used for final diagnosis. Cases with incomplete information, or inadequate radiographs were excluded from further analysis. In this 14-year time period 1,500 cases were identified for review among the approximately 7700 cases referred during that time period.

Each case was classified by radiographic diagnosis and histology when available. Histology was used for diagnosis when radiographs were not interpretable due to disruption of the fetal skeleton, or if the radiographic findings suggestive a novel pattern of abnormalities. Diseases or diagnosis categories followed the nosology established by the International Nosology Committee [Superti-Furga and Unger, 2007]. For this analysis, disorders were also grouped into the following categories; osteogenesis imperfecta types 2 , thanatophoric dysplasia, achondrogenesis type 2, campomelic dysplasia, short-rib polydactyly syndromes, other specific skeletal dysplasias, unclassified skeletal dysplasias, genetic syndromes, and normal. The classification "normal" was used if the radiographs failed to show any specific skeletal abnormalities. Each case was placed into the above listed categories to determine their relative frequency using the total number of cases for the time period. In addition, for each case and category, the radiographic diagnosis was compared to the referring ultrasound diagnosis to determine percent correlation for ultrasound versus radiographic diagnosis.

\section{Prospective Analysis}

From the period 1996-2006 the International Skeletal Dysplasia Registry (ISDR) analyzed prenatal-onset skeletal dysplasias by reviewing consecutively referred ultrasound images in 500 cases (1438 weeks gestation). Each referred case (real-time ultrasound or hard-copy ultrasound images) were reviewed or performed by one of the authors (DK). Analysis and documentation of real-time ultrasound included the following parameters found to be important for diagnosis: appearance, shape and size 
of the cranium, measurements of the mandible and clavicles, measurements of all long bones, chest circumference and abdominal circumference. Other parameters that were evaluated that aided in differentiating these disorders included the shape of the scapula, vertebral bodies and ribs, the mineralization and shape of the long bones, the appearance of the metaphyseal ends of the bones, presence of facial dysmorphism, evaluation of the hands and feet (absent or extra digits), and presence or absence of the calcaneus and distal femur epiphyses in the late second and third trimester, as well as other congenital anomalies, posturing of the distal extremities and neck and amniotic fluid volume.

Post-delivery radiographs and, when available, histomorphology, were obtained for final diagnosis. Only cases with available radiographs were included in the analysis. As described for the retrospective analysis, each case was analyzed to determine the relative frequency of the disorder and the correlation between prenatal ultrasound versus radiographic diagnosis. Cases with only preliminary, ultrasoundbased diagnoses were classified in the results section as "lost-to-follow-up."

\section{RESULTS}

\section{Retrospective Analysis}

From 1990 to 2004, the International Skeletal Dysplasia Registry (ISDR) received clinical information, radiographs and histology on 1,500 cases of prenatal-onset skeletal dysplasias. The gestational ages when the fetal skeletal abnormality was recognized by ultrasound are shown in Figure 1. The majority of cases (70\%) were recognized in the second trimester and many of these prior to 20 weeks gestation. For the remaining 30\% of the cases, the skeletal dysplasia was recognized by ultrasounds performed after 24 weeks. In approximately $4 \%$ of the cases, gestational age at time of diagnosis was not available.

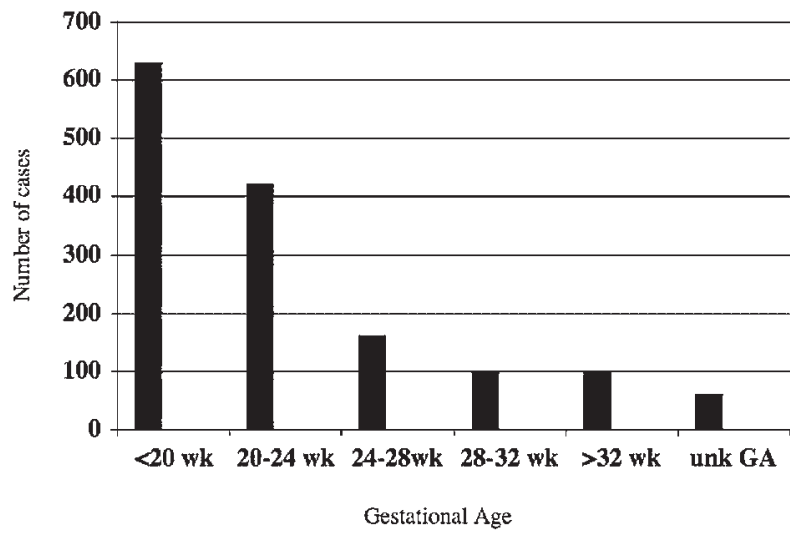

FIG. 1. Bar graph depicting the gestational age distribution at which prenatal onset skeletal dysplasias were detected by ultrasound.
For the 1,500 retrospective cases reviewed, the referring diagnoses are noted in Figure 2A. The majority of cases (67\%) were referred utilizing the diagnosis, "skeletal dysplasia," with no specific disorder indicated. The other most commonly used referring diagnoses were thanatophoric dysplasia (13\%), osteogenesis imperfecta (10\%), campomelic dysplasia (6\%), and short-rib polydactyly (4\%). Among the entire cohort of cases, subsequent radiographic and histologic analysis allowed a definitive diagnosis to be made in $95 \%$ of the cases: thanatophoric dysplasia (11\%), osteogenesis imperfecta type 2 (20\%), achondrogenesis type $2(8.2 \%)$, campomelic dysplasia (3.6\%), other distinct skeletal dysplasias (35.7\%), unclassified skeletal dysplasias (4.5\%), genetic syndromes (12\%) and normal (5\%) (Fig. 2B).

The referring ultrasound and final radiographic diagnoses were the same in $41.7 \%$ of the cases and correlations between ultrasound versus radiographic diagnoses are depicted in Figure 3. Osteogenesis imperfecta type 2, thanatophoric dysplasia and achondrogenesis 2 accounted for almost $40 \%$ of the prenatal-onset osteochondrodysplasias in this cohort and the correlation between referral ultrasound diagnosis and final radiographic diagnosis in these more commonly seen skeletal disorders was approximately $50 \%$. However, the referring diagnosis accuracy by ultrasound for the "other distinct skeletal dysplasia category" only approached $28 \%$.

\section{Prospective Analysis}

In the prospective analysis of 500 consecutive cases, our review of the images yielded a preliminary
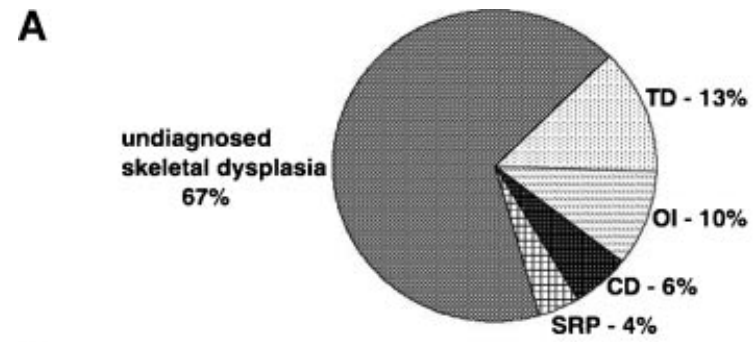

B

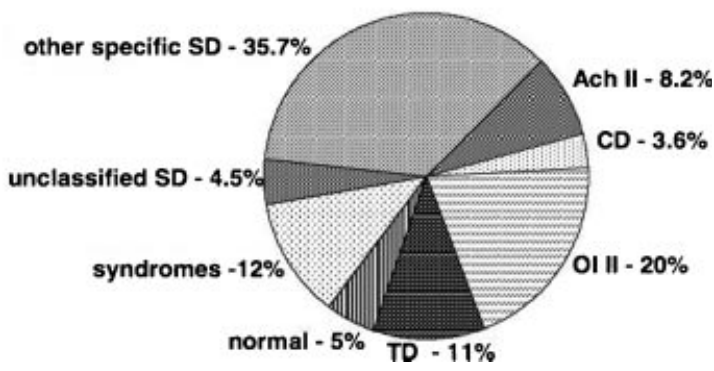

FIG. 2. A: Pie chart showing the referring diagnoses of 1,500 prenatal-onset skeletal dysplasia cases in the retrospective analysis based on prenatal diagnoses referred to the ISDR (International Skeletal Dysplasia Registry). B: Pie chart of the final diagnoses determined by radiographs and histology when available, of 1,500 prenatal-onset skeletal dysplasias. TD, thanatophoric dysplasia; OI, osteogenesis imperfecta type II; CD, campomelic dysplasia; SRP, short-rib polydactyly; ACH II, achondrogenesis II; SD, skeletal dysplasias. 
1920

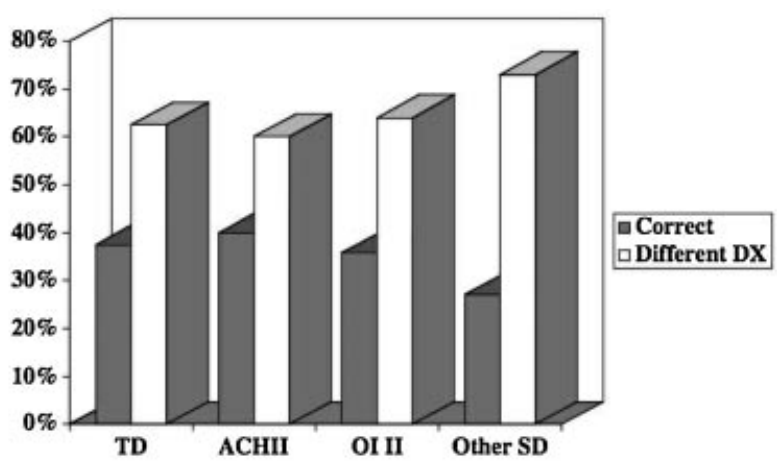

FIG. 3. Bar graph showing the percent correlations between prenatal ultrasound versus radiographic diagnoses for specific skeletal dysplasias in the retrospective analysis. TD, thanatophopric dysplasias, ACH II, achondrogenesis 2, OI II, osteogenesis imperfecta type 2, other SD, other skeletal dysplasias.

ultrasound diagnosis in $92 \%$ of cases, while in the remaining $8 \%$ (40 cases) no definitive diagnosis or differential diagnosis could be made. In this cohort, postnatal radiographs were obtained in 405 cases (81\%). Since no final radiographs were available to make a final definitive diagnosis in 95 cases, these cases were excluded from further analysis. Comparisons made between our preliminary ultrasound diagnosis and final radiographic diagnosis showed that they were identical in $78 \%$ of the cases. Among the cases where lethality was predicted based on chest size and configuration, lethality was accurately predicted in $96.8 \%$ of cases. In this study, the definition of lethality utilized was that without heroic measures the infant would die in the first few weeks of life.
RAKOW ET AL.

Categorization of the 405 cases by disease identified the relative frequency for the three most common disorders as osteogenesis imperfecta type 2 (16\%, 65 cases), thanatophoric dysplasia (12\%, 50 cases), and achondrogenesis type 2 (6\%, 24 cases). These percentages were similar to those obtained from the much larger retrospective analysis and they accounted for slightly greater than one-third of the prenatal-onset skeletal dysplasias referred to the ISDR. These three lethal disorders, all share small chest circumferences with abnormal chest to abdominal circumference ratios. However, they can be differentiated based on the on several ultrasound findings. In osteogenesis imperfecta type 2, the abnormal ultrasound findings included: under-mineralization of the calvarium, flattened profile, fractures with asymmetric appearing long bones, beaded (callus formation) ribs, and hands appearing grossly normal (Fig. 4). Thanatophoric dysplasia was characterized by relative macrocephaly with narrowed or closed cranial sutures, metopic bossing on profile, very shortened, symmetric long bones that were either bowed or straight, present but flattened vertebral bodies, very short hands and digits and no delay in the secondary ossification centers (Fig. 5). Achondrogenesis 2 was frequently associated with cystic hygromas or very large nuchal lucencies, a flattened facial profile with micrognathia, almost absent mineralization of the vertebral bodies, very short appendicular long bones with metaphyseal spikes and very small hands and feet (Fig. 6). Among the other $2 / 3$ of the 405 cases, the final radiographic diagnoses varied and Table I catalogs the heterogeneity of the skeletal
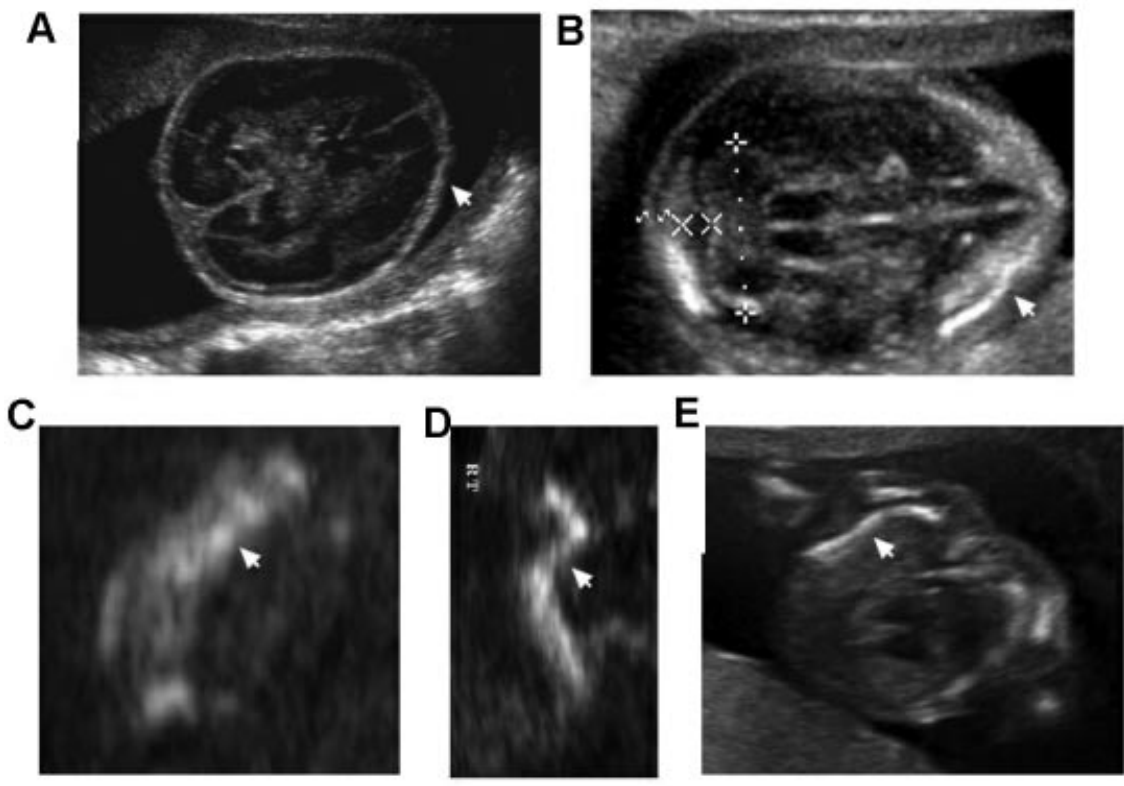

FIG. 4. Sonographic images of osteogenesis imperfecta type 2 (OI type 2) showing abnormal findings. A: Absent mineralization of the calvarium (18 weeks gestation). B: Diminished mineralization of the calvarium (16 weeks gestation); arrow points to an area of calvarium mineralization. C: Femur (18 weeks gestation) showing bright areas (echodense) representing fracture and callus formation (arrow). D: Femur (16 weeks gestation) showing irregular bending (arrow). E: Saggital chest view in OI type 2 showing wavy or S-shaped ribs (arrow) and ribs that do not surround two-thirds of the chest circumference. 


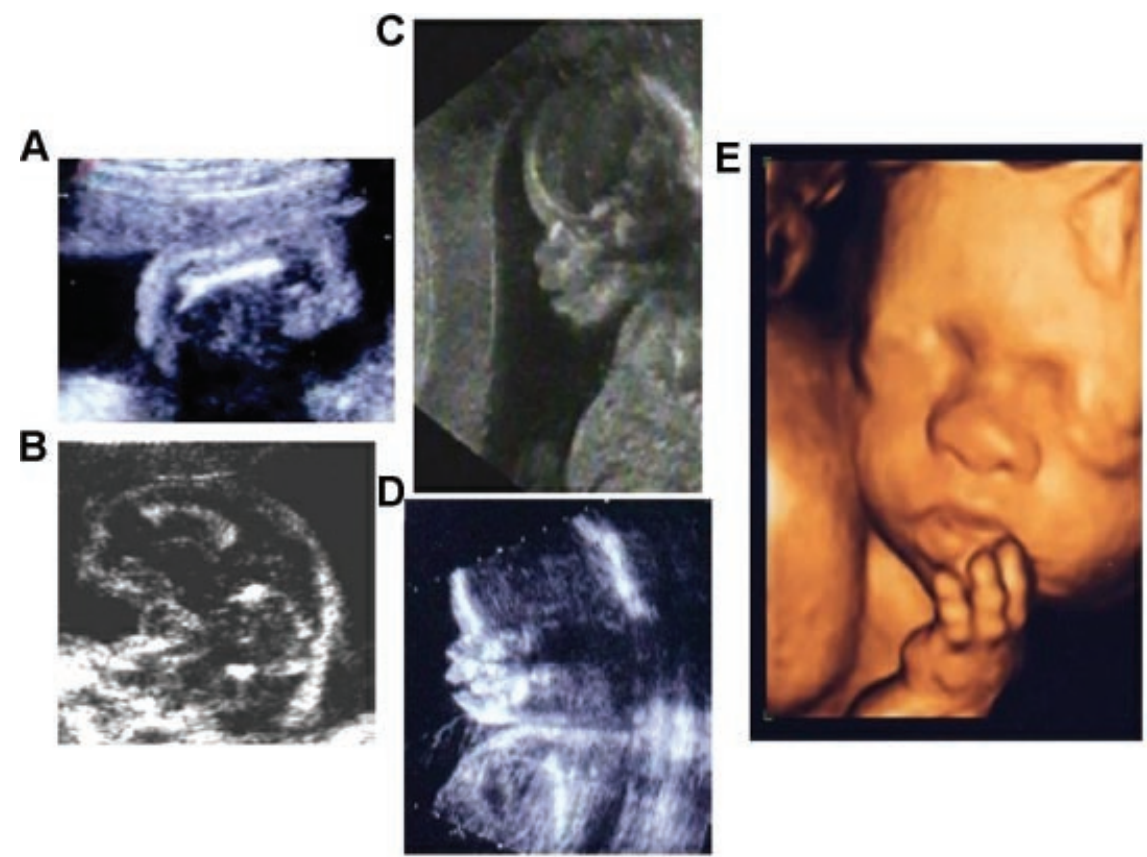

FIG. 5. Sonographic images of thanatophoric dysplasia. A: Femur (16 weeks gestation) in thanatophoric type 2 (TD 2 ) is straight and very short with metaphyseal spikes. B: Femur (20 weeks gestation) in thanatophoric type I (TD1) is curved with metaphyseal flaring. C.D: Two dimensional sonogram of a fetus with thanatophoric type I (TD1) (18 weeks gestation) with a tall/high metopic ridge, almost absent nasal bone (D), and facial disproportion since the metopic suture and mandible are not in the same plane, suggesting coronal synostosis. E: Three dimensional sonographic image of TD1 at 24 weeks showing a flattened nasal bridge and brachydactyly.

dysplasias and dysostoses that can be visualized in the prenatal period.

\section{DISCUSSION}

The skeletal dysplasias have been estimated to account for about $5 \%$ of the genetic disorders seen in the newborn period [Orioli et al., 1986] and represent a significant burden to many families because of potential lethality, and short- and long-term medical complications, and recurrence risk. Therefore, informing parents and physicians caring for these patients with accurate diagnoses and natural history information is critical to providing the best care for
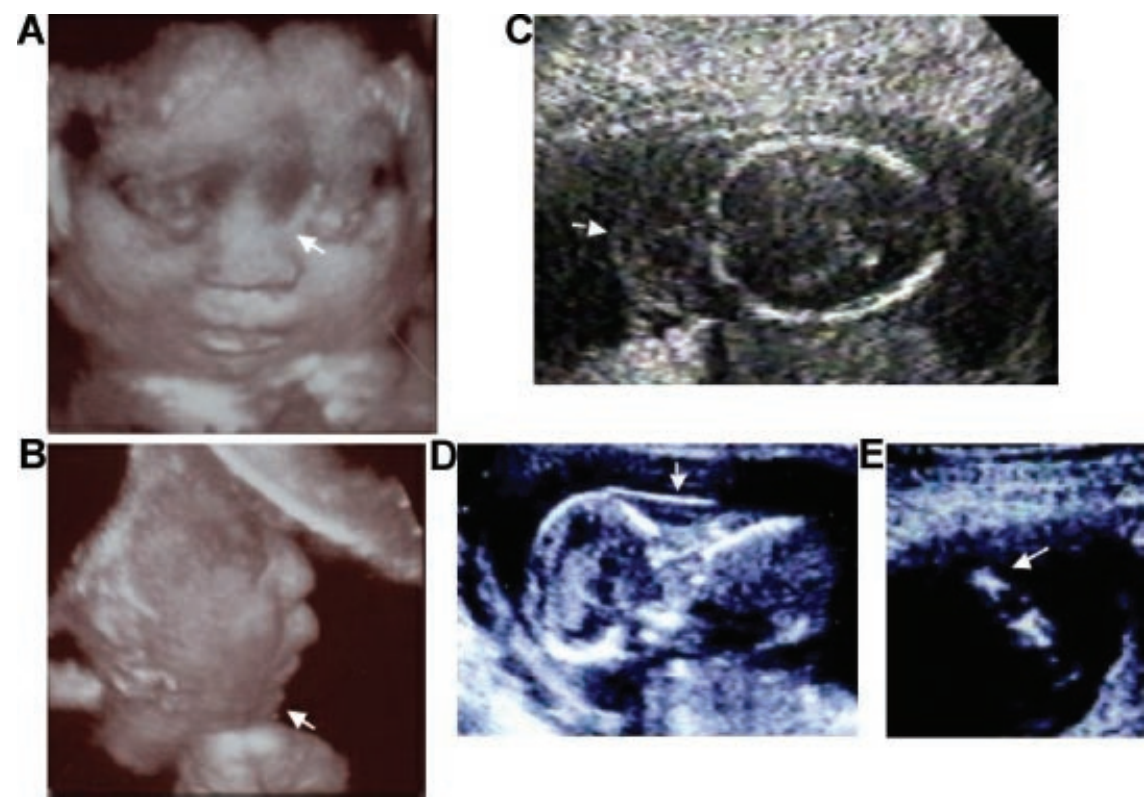

FIG. 6. Sonographic images of achondrogenesis type 2. A,B: Three dimensional sonographic image of a fetus with achondrogenesis type 2 at 22 weeks gestation showing severe by flattened nasal bridge (arrow) and micrognathia (arrow). C: Cystic hygroma (arrow) at 20 weeks gestation. D: Increased nuchal fold (12 weeks gestation) (arrow) in the same fetus from (C). E: Femur (16 weeks gestation) showing severe micromelia and metaphseal spikes. 
TABLE I. Skeletal Disorders Identified in the Prenatal Period

Acheiropody

Achondrogenesis 1B

Achondrogenesis 2

Achondroplasia

Acromandibular syndrome

Apert syndrome

Asphyxiating thoracic dystrophy

Atelosteogenesis types 1, 2, and 3

Barnes syndrome

Brachydactyly B

Caffey disease-perinatal type

Campomelic dysplasia

CHILD syndrome

Chondrodysplasia Punctata-rhizomelic type

Chondrodysplasia Punctata-Conradi-Hunerman

Cleidocranial dysplasia

Desbuquois dysplasia

Diastrophic dysplasia

Ectrodactyly syndrome

Ehlers-Danlos syndrome type 7B

Ellis van Creveld syndrome

Focal femoral hypoplasia with/without unusual facies

Gracile bone dysplasia

Hypochondrogenesis

Hypophosphatasia-perinatal lethal type

Kniest dysplasia

Larsen syndrome

Lethal Platyspondylic dysplasia-Torrance type

Metaphyseal Chondrodysplasia-McKusik type

Metatropic dysplasia

Mucolipidosis 2

Multiple synostosis syndrome

Nail-Patella syndrome

Neu-Laxova syndrome

OSMED dysplasia

Opsismodysplasia

Osteogenesis Imperfecta type 2 and 3

Otopalatodigital syndrome type 2

Pfeiffer syndrome

Pseudodiastrophic dysplasia

Roberts SC Phocomelia

SMD-Sedaghatian type

Schneckenbecken dysplasia

Short-rib polydactyly syndrome-types 2, 3, and 4

Spondyloepiphyseal dysplasia congenita

Spondylothoracic dysplasia

Stuve-Wiedemann syndrome

Thanatophoric dysplasia 1 and 2

Tibia-Ectrodactyly syndrome

Type 2 hemimelia

Unclassified skeletal dysplasias

these patients. The routine use of prenatal ultrasound has dramatically increased in the last three decades and has become widely accepted as a means of diagnosing congenital anomalies. This was reflected in the retrospective analysis, which showed an increasing number of cases referred on an annual basis. Because the fetal skeleton is well visualized by ultrasound, the diagnostic accuracy of this modality should be high for skeletal disorders. However, most series note a diagnostic accuracy for the skeletal dysplasias at less than 50\% [Donnenfeld and Mennuti, 1987; Sharony et al., 1993; Rasmussen et al., 1996; Gaffney et al., 1998; Tretter et al., 1998; Doray et al., 2000; Parilla et al., 2003]. Because some of these series are from the late 1980s and technical improvements have subsequently been made in ultrasound imaging, to make the variable of inferior equipment less relevant, we chose to only analyze cases from 1990 to 2004 in the retrospective analysis. Even with this selection, the 1,500 cases in the retrospective analysis showed a diagnostic accuracy of $41 \%$.

The reasons for the low diagnostic accuracy in referring ultrasound prenatal diagnosis for the osteochondrodysplasias are varied. There are over 350 clinically distinct skeletal dysplasias and even more dysostoses syndromes, and they all occur rarely. Table I illustrates the large number of distinct disorders that were seen in the prenatal period. Few centers may have the clinical experience to differentiate all these disorders. Similar to the characteristic radiographic findings in these disorders, the sonographic findings can vary with gestational age. For example, in this cohort, cloverleaf skull (kleebattschädel), which is a characteristic finding in some craniosynostosis disorders, was not seen before 1618 weeks gestation. The skeletal elements also undergo mineralization at different gestational ages, and ultrasound findings based on the appearance of specific bones that lead to diagnostic clues may not be apparent at certain gestational ages. The earliest secondary epiphysis seen by sonogram is the calcaneus appearing at approximately 20 weeks. Thus, attempting to diagnose epiphyseal delay prior to that gestational age is not possible. In many of the nonlethal skeletal dysplasias, such as achondroplasia and spondyloepiphyseal dysplasia congenita (SEDC), the fall-off in long bone measurements (below the 5th centile) was not observed until the second half of the second trimester (18 weeks). Therefore in addition to recognizing the characteristic sonographic findings in each skeletal dysplasia, prenatal diagnostic centers need to know the gestational age at which the skeletal abnormalities appear on sonogram.

To address whether the diagnostic accuracy of ultrasound could be improved upon, a prospective analysis of the prenatal diagnosis of the skeletal dysplasias was performed by analyzing the ultrasound findings from referred cases. The diagnostic criteria used were based on a clinical and radiographic approach. The diagnostic accuracy could be improved to almost $80 \%$ by analyzing each case for the known radiographic findings in individual skeletal dysplasias. The sonographic findings were categorized based on the components of the appendicular and axial skeleton (spondylo-, rhizo-, meso-, and acro- segments) that were abnormal. The findings were then categorized into specific groups of skeletal dysplasias, for example, a spondlyometaphyseal dysplasia, and then the diagnosis was refined within that radiographic group [Spranger et al., 2002; Lachman, 2007] by differentiating the disorders within that diagnostic category group. Not 
only did this approach lead to increased diagnostic accuracy, it also provided a differential diagnosis list for prognostic and genetic counseling purposes.

Further comparisons noted from the prospective analysis showed that many of the referring centers did not obtain ultrasound measurements of the chest circumference or lateral views of the chest for configuration. Without these views, it is very difficult to distinguish lethal from non-lethal skeletal dysplasias. By determining which skeletal elements are abnormal, and if either the configuration or size of the chest suggests lethality, then a more accurate diagnosis can be made. If the specific diagnosis or differential diagnosis list predominantly includes skeletal dysplasias known to be lethal by natural history, the families can be more accurately informed.

Detailed analysis of the hands, feet and facies were also useful in refining the differential diagnosis. Evaluation of the hands and feet for brachydactyly or abnormal configuration/posturing was extremely helpful and allowed for improved diagnostic accuracy. In addition, the facies in the skeletal dysplasias are frequently dysmorphic and many of the findings, such as frontal bossing, hypertelorism, midface hypoplasia and micrognathia, are readily visualized by sonogram [Krakow et al., 2003]. Evaluation of the distal extremities and the facies, in addition to the skeleton, provides information that allows ultrasonographers and clinical geneticists to group together a constellation of findings to determine the diagnosis.

Perhaps another reason for the difficulty in prenatal diagnosis of the skeletal dysplasias is due to the variability in appearance of ultrasound findings in individual skeletal dysplasias. As commonly seen in their clinical presentation and radiographic findings, there can be marked variability within each phenotype. The cases studied represented a wide spectrum of disease severity. For example, there were cases of osteogenesis imperfecta type 2 with a single fracture at initial evaluation and others with crumpled long bones. There were cases of campomelic dysplasia with and without campomelia. This variability in appearance may explain why the referring diagnostic accuracy for even the more common skeletal dysplasias, that is, thanatophoric dysplasia, osteogenesis imperfecta type 2 and achondrogenesis 2 only approached $50 \%$. Thus, in addition to knowledge regarding the specific radiographic and clinical features of these disorders, clinicians need to be aware of the varied phenotypic manifestations in the fetal period.

The retrospective analysis allowed us to determine the relative frequency of some individual skeletal dysplasias. One of the potential limitations of this study is ascertainment bias in the selection of cases referred to the ISDR. However, there were 1,500 cases in the retrospective study, and the prospective analysis yielded similar findings, so some conclusions can be drawn. The most commonly seen disorders were osteogenesis imperfecta (20\%), thanatophoric dysplasia (11\%), and achondrogenesis type II (8.2\%). This confirms the data obtained by Stoll et al. [1989] and Orioli et al. [1986] on the birth prevalence of skeletal dysplasias, which noted that these were the most prevalent skeletal dysplasias, though our frequencies vary slightly. In both of these studies, achondroplasia was also noted to have high birth prevalence. In both our retrospective and prospective cohorts there were some cases of achondroplasia, however they were not as common as thanatophoric dysplasia, osteogenesis imperfecta type 2 and achondrogenesis type 2 . This bias may be due to two factors: one, many cases of achondroplasia are not recognized by prenatal diagnosis, especially if the ultrasound was performed in the mid-second trimester, and two, perhaps recognized cases of this common disorder are not as frequently referred to the ISDR for consultation. Evaluation of the cases of achondroplasia (second trimester) seen in the prospective study showed that there were some clues to diagnosis. These included relative macrocephaly when compared to the femur lengths (usually the femur lengths were 5th centile and the head circumference was 90th centile or above), metopic bossing, and widening of the distal femur with normal secondary ossification centers. In addition to the disorders listed above, campomelic dysplasia was seen in $4 \%$ of our cases. Interestingly, when comparing the referring diagnoses to final diagnoses, this disorder was associated with the highest diagnostic accuracy in the prenatal period (50\%). This is probably due to the characteristic angulated femora seen on ultrasound in many cases of campomelic dysplasia. Among the remaining cases of campomelic dysplasia, osteogenesis imperfecta type 2 was the most common referring ultrasound diagnosis. Evaluation of the configuration of the scapula (hypoplastic in campomelic dysplasia) and profile (micrognathia in campomelic dysplasia) can help differentiate these disorders.

There were cases in both the retrospective and prospective analyses for which no conclusive diagnosis could be made and these "unclassified" skeletal dysplasias accounted for 5\% of the cases. Approximately $12 \%$ of the cases reviewed were dysmorphic syndromes with skeletal anomalies. Only about $20 \%$ of those cases were correctly assessed in the prenatal period. In 5\% of the cases seen, a skeletal dysplasia was suspected, yet the radiographic findings were normal. We surmise that these cases either represent early-onset intrauterine growth retardation or significant biologic variability in long bone length. This highlights the importance of a full evaluation of the fetal axial and appendicular skeleton, including the size and shape of the long bones, epiphyseal centers and relative proportions 
to differentiate skeletal dysplasias from growth restriction (suggested fetal ultrasound evaluation for a suspected fetal skeletal dysplasia is listed at http://www.cedars-sinai.edu/9934.html).

One of the concerning findings in the prospective analysis was that for $18 \%$ of the 500 cases, no final diagnosis could be made because post-delivery radiographs and autopsy were not obtained. Although these cases were excluded from the analysis, absence of post-delivery radiographs can compromise patient care because of the inability to confirm the sonographic diagnoses and provide recurrence risk. Since a firm diagnoses cannot always be made on the basis of ultrasound findings alone, radiographic (autopsy) and histologic information remain an irreplaceable component in defining diagnoses and providing accurate counseling for families.

With the increasing use of prenatal sonogram for the detection of congenital anomalies, there has been increased detection of prenatal-onset skeletal dysplasias. The detailed approach to prenatal sonogram described in the prospective analysis demonstrates that this approach can lead to continued improvement in the diagnostic accuracy of these disorders in the prenatal period. The coordinated interaction between prenatal diagnosticians, geneticists, radiologists, and scientists with focused expertise in these disorders will continue to lead to improvements in the care of these patients and their families.

\section{ACKNOWLEDGMENTS}

The authors would like to thank the families and referring health care providers who participated in this study. DLR, RSL, WRW, DK are supported by NIHCD HD2257 and DK is supported by the Joseph Drown Foundation and the NIH-sponsored General Clinical Research Center Grant (M01-RR00425) and DK and WRW are Winnick Family Scholar awardees.

\section{REFERENCES}

Bahado-Singh RO, Raymond M. 2007. The 20-week scan: Beyond biometry and anatomy. Clin Obstet Gynecol 50:478-486.

Donnenfeld AE, Mennuti MT. 1987. Second trimester diagnosis of fetal skeletal dysplasias. Obstet Gynecol Surv 42:199-217.

Doray B, Favre R, Viville B, Langer B, Dreyfus M, Stoll C. 2000. Prenatal sonographic diagnosis of skeletal dysplasias. A report of 47 cases. Ann Genet 43:163-169.

Eames BF, de la Fuente L, Helms JA. 2003. Molecular ontogeny of the skeleton. Birth Defects Res C Embryo Today 69:93-101.

Gaffney G, Manning N, Boyd PA, Rai V, Gould S, Chamberlain P. 1998. Prenatal sonographic diagnosis of skeletal dysplasiasA report of the diagnostic and prognostic accuracy in 35 cases. Prenat Diagn 18:357-362.

Hall CM. 2002. International nosology and classification of constitutional disorders of bone (2001). Am J Med Genet 113:65-77.

Krakow D, Williams J, Poehl M, Rimoin DL, Platt LD. 2003. Use of three-dimensional ultrasound imaging in the diagnosis of prenatal-onset skeletal dysplasias. Ultrasound Obstet Gynecol 21:467-472

Kuller JA, Katz VL, Wells SR, Wright LN, Mc Mahon MJ. 1996. Cesarean delivery for fetal malformations. Obstet Gynecol Survey 51:371-375.

Lachman RS. 2007. Taybi and Lachman's radiology of syndromes, metabolic disorders and skeletal dysplasias. Philadelphia: Mosby-Elsevier.

Larson WJ. 1993. Human embryology. NewYork: Churchill Livingstone. p 281-310.

Mundlos S. 2001. Defects of human skeletogenesis-Models and mechanisms. Novartis Found Symp 232:81-91.

Olsen BR, Reginato AM, Wang W. 2000. Bone development. Annu Rev Cell Dev Biol 16:191-220.

Orioli IM, Castilla EE, Barbosa-Neto JG. 1986. The birth prevalence rates for the skeletal dysplasias. J Med Genet $23: 328-332$

Parilla BV, Leeth EA, Kambich MP, Chilis P, MacGregor SN. 2003. Antenatal detection of skeletal dysplasias. J Ultrasound Med $22: 255-258$

Rasmussen SA, Bieber FR, Benacerraf BR, Lachman RS, Rimoin DL, Holmes LB. 1996. Epidemiology of osteochondrodysplasias: Changing trends due to advances in prenatal diagnosis. Am J Med Genet 61:49-58.

Saltvedt S, Almström H, Kublickas M, Valentin L, Grunewald C. 2006. Detection of malformations in chromosomally normal fetuses by routine ultrasound at 12 or 18 weeks of gestationA randomised controlled trial in 39,572 pregnancies. Br J Obstet Gynaecol 113:664-674.

Savarirayan R. 1999. Common phenotype and etiology in warfarin embryopathy and X-linked chondrodysplasia punctata (CDPX). Pediatr Radiol 29:322.

Shanske AL, Bernstein L, Herzog R. 2007. Chondrodysplasia punctata and maternal autoimmune disease: A new case and review of the literature. Pediatrics 120:e436e441.

Sharony R, Browne C, Lachman RS, Rimoin DL. 1993. Prenatal diagnosis of the skeletal dysplasias. Am J Obstet Gynecol 169:668-675.

Shum L, Coleman CM, Hatakeyama Y, Tuan RS. 2003. Morphogenesis and dysmorphogenesis of the appendicular skeleton. Birth Defects Res C Embryo Today 69:102-122.

Spranger JW, Brill PW, Poznaski A. 2002. Bone dysplasias. New York: Oxford University Press.

Stoll C, Dott B, Roth MP, Alembik Y. 1989. Birth prevalence rates of skeletal dysplasias. Clin Genet 35:88-92.

Superti-Furga A, Unger S. 2007. Nosology and classification of genetic skeletal disorders: 2006 revision. Am J Med Genet Part A $143 \mathrm{~A}: 1-18$.

Superti-Furga A, Bonafé L, Rimoin DL. 2001. Molecular-pathogenetic classification of genetic disorders of the skeleton. Am J Med Genet 106:282-293.

Sutton VR, McAlister WH, Bertin TK, Kaffe S, Wang JC, Yano S, Shaffer LG, Lee B, Epstein CJ, Villar AJ. 2003. Skeletal defects in paternal uniparental disomy for chromosome 14 are recapitulated in the mouse model (paternal uniparental disomy 12). Hum Genet 113:447-451.

Tretter AE, Saunders RC, Meyers CM, Dungan JS, Grumbach K, Sun CC, Campbell AB, Wulfsberg EA. 1998. Antenatal diagnosis of lethal skeletal dysplasias. Am J Med Genet 75:518-522.

van Zalen-Sprock RM, Brons JT, van Vugt JM, van der Harten HJ, van Geijn HP. 1997. Ultrasonographic and radiologic visualization of the developing embryonic skeleton. Ultrasound Obstet Gynecol 9:392-397.

Walter CA, Shaffer LG, Kaye CI, Huff RW, Ghidoni PD, McCaskill C, McFarland MB, Moore CM. 1996. Short-limb dwarfism and hypertrophic cardiomyopathy in a patient with paternal isodisomy 14: 45,XY,idic(14)(p11). Am J Med Genet 65: $259-265$. 